\title{
Síndrome pseudobulbar en pacientes con accidente cerebrovascular en Argentina
}

\author{
Matías Alet ${ }^{1}$, Constanza Segamarchi ${ }^{1}$, Santiago Claverie ${ }^{1}$, \\ Leonardo González ${ }^{1}$, Sandra Lepera ${ }^{1}$, Raúl Rey ${ }^{1}$
}

1. Sección de Enfermedades Cerebrovasculares, Servicio de Neurología. Hospital "J. M. Ramos Mejía”, Ciudad de Buenos Aires, Argentina.

Autor correspondiente: Matías Javier Alet, E-mail: matias.alet@gmail.com

\begin{abstract}
Resumen
Introducción y objetivo: El síndrome pseudobulbar se define por episodios involuntarios o exagerados de risa o llanto, posteriores a una enfermedad específica. El síndrome pseudobulbar después del accidente cerebrovascular se reporta entre el 11\%-34\% de los pacientes. En nuestra población está subdiagnosticado. Determinamos su frecuencia en un grupo de pacientes post-ACV. Pacientes y métodos: Estudio observacional prospectivo desde junio/2017 a junio/2018. Se evaluaron dos poblaciones: ACV crónico (Grupo A) y ACV agudo (Grupo B). Se excluyeron pacientes con enfermedad psiquiátrica grave, deterioro cognitivo y/o afasia. Se realizaron las escalas PLACS ("pathological laughing and crying scale") y CNS-LS ("Center for Neurologic Study-Lability Scale"). El síndrome pseudobulbar se definió por criterios diagnósticos más ambas escalas positivas. Resultados: Cincuenta pacientes fueron evaluados. El 68\% eran hombres. Edad media: $65 \pm 12$ años. Grupo A: 19 casos y grupo B: 31 casos. PLACS positiva: $21 \%$ grupo A y $29 \%$ grupo B. CNS-LS positiva: $58 \%$ grupo A y $26 \%$ grupo B. Ambas escalas positivas en 8 pacientes ( 2 en crónico y 6 en agudo). Tres de ellos tenían depresión asociada. Conclusiones: El síndrome pseudobulbar debe examinarse cuidadosamente luego del ACV. La depresión post-ACV debe diferenciarse de la labilidad emocional por síndrome pseudobulbar. La administración de una sola escala no sería suficiente para la orientación diagnóstica.
\end{abstract}

Palabras claves: Accidente Cerebrovascular - Labilidad emocional - Síndrome pseudobulbar.

\section{PSEUDOBULBAR AFFECT IN STROKE PATIENTS IN ARGENTINA}

\begin{abstract}
Background: Pseudobulbar affect is defined by involuntary or exaggerated episodes of laughter or crying, subsequent to a specific disease. After-stroke pseudobulbar affect is reported in 11\%-34\% of patients. In our population is underdiagnosed. Our objective was to determine its frequency in a group of stroke patients. Patients and methods: Prospective observational study from June/2017 to June/2018. Two populations were evaluated: chronic stroke (Group A) and acute stroke (Group B). Patients with severe psychiatric illness, cognitive impairment and/or aphasia were excluded. PLACS ("pathological laughing and crying scale") and CNS-LS ("lability scale for pseudobulbar affect") scales were performed. Pseudobulbar affect was defined with diagnostic criteria plus both positive scales. Results: Fifty patients were evaluated. Sixty-eight percent were men. Mean age: 65 \pm 12 years. Group A: 19 cases and group B: 31 cases. Positive PLACS: 21\% group A and 29\% group B. Positive CNS-LS: 58\% group A and 26\% group B. Both positive scales in 8 patients ( 2 in group A and 6 in group B). Three of them had associated depression. Conclusions: Pseudobulbar affect should be screened after stroke. Post-stroke depression is frequent and differential diagnose with emotional lability attributable to pseudobulbar affect must be ruled out. The administration of a single scale would not be sufficient for diagnostic guidance.
\end{abstract}

Keywords: Stroke - Emotion incontinence - Pseudobulbar affect. 


\section{Introducción}

El accidente cerebrovascular (ACV) sigue siendo una causa principal de discapacidad a largo plazo en todo el mundo (1). Habitualmente se presta mayor atención a las secuelas motoras e impedimentos físicos. Sin embargo, las discapacidades neuropsiquiátricas también impactan negativamente en la calidad de vida del paciente. Una de esas complicaciones psicosociales del ACV se conoce comúnmente como incontinencia o labilidad emocional, o propiamente como síndrome pseudobulbar (SPB). Este síndrome se define como la incapacidad de expresar adecuadamente las emociones a través de gestos verbales y no verbales. La expresión facial de las emociones más básicas, como la felicidad o la tristeza, requiere una interacción compleja de varias regiones del cerebro, y la interrupción a cualquier nivel de esta red puede conducir a una condición de labilidad emocional y desregulación afectiva (2). Los pacientes con SPB tienen menos control sobre sus afectos, que están notablemente exagerados o inapropiados. Esto crea una tremenda carga psicosocial para los pacientes y cuidadores, conduce a situaciones embarazosas, con disminución en las interacciones sociales y repercusión negativa en su calidad de vida (3).

En Argentina, no hay información sobre este tipo de discapacidad. Por lo tanto, se decidió estudiar una cohorte de pacientes con ACV y evaluar la presencia de SPB en nuestra población.

\section{Pacientes y métodos}

Se realizó un estudio observacional prospectivo. Se incluyeron pacientes con diagnóstico de ACV isquémico o hemorrágico, en el período comprendido entre junio de 2017 y junio de 2018. Se excluyeron los pacientes con enfermedad psiquiátrica severa, deterioro cognitivo severo y/o afasia.

La población se dividió en el Grupo A: ACV crónico, entre 3 y 12 meses (que acudieron a la clínica ambulatoria de $\mathrm{ACV}$ ); y Grupo B: ACV agudo (que ingresaron en la Unidad de ACV de nuestro centro y fueron evaluados en la primera semana desde el inicio de los síntomas).

En la primera entrevista, se informó a los pacientes de las características y objetivos del estudio, y se obtuvo consentimiento informado por escrito en todos los casos. Se evaluaron los datos demográficos y antecedentes médicos. Los ACV se diferenciaron entre isquémicos o hemorrágicos. Los ACV isquémicos se clasificaron según los criterios de TOAST y la gravedad se definió mediante la escala NIHSS. El territorio vascular afectado se dividió en supratentorial/infratentorial y anterior/posterior.

Se evaluó en cada paciente, familiares y/o cuidadores la manifestación en forma espontánea y luego se indagó en forma dirigida por síntomas compatibles con SPB. Se realizaron las escalas PLACS ("Pathological Laughing and Crying Scale") y CNS-LS ("Center for Neurologic StudyLability Scale"). PLACS se consideró positiva si el paciente obtuvo 13 puntos o más. CNS-LS se consideró positiva si el paciente obtuvo 13 puntos o más. El SPB se consideró presente en aquellos pacientes que cumplieran con los criterios diagnósticos propuestos (Tabla 1) (4) y puntuaran ambas escalas positivas.

Tabla 1. Criterios diagnósticos propuestos para síndrome pseudobulbar $\left(^{*}\right)$

\section{Criterios esenciales}

- El paciente experimenta episodios de expresión emocional involuntaria o exagerada, que incluye risa, llanto u otras manifestaciones emocionales.

- Los episodios representan un cambio en la reactividad emocional habitual del paciente, son exagerados

o incongruentes con el estado emocional subjetivo y son independientes o excesivos en relación el estímulo desencadenante.

- Los episodios causan malestar clínico significativo, o discapacidad social y/o laboral.

- Los síntomas no pueden atribuirse a otros trastornos neurológicos o psiquiátricos, 0 al efecto de sustancias psicoactivas.

\section{Criterios secundarios}

- Los pacientes pueden experimentar cambios autonómicos (por ejemplo, enrojecimiento facial) y signos pseudobulbares (por ejemplo, aumento del tono mandibular, reflejo nauseoso aumentado, debilidad de la lengua, disartria y disfagia).

- Los pacientes pueden mostrar propensión a la ira.

$\left(^{*}\right)$ Criterios diagnósticos adaptados de Miller A, Pratt H, Schiffer RB. Pseudobulbar affect: The spectrum of clinical presentations, etiologies and treatments. Expert Rev Neurother 2011;11(7):1077-88 
Se administró el inventario de depresión de Beck (IDB) para descartar depresión asociada. Una puntuación mayor o igual a 13 se consideró como un punto de corte para el diagnóstico de depresión. No se consideró presencia de SPB en pacientes con depresión.

El estudio fue presentado y aprobado por el Comité de Ética del Hospital General "J. M. Ramos Mejía”.

El software IBM SPSS Statistics 22.0 se utilizó para analizar los resultados. Las variables categóricas se presentan como números o porcentajes. Las variables cuantitativas se expresan como medias y desviaciones estándar (DE), o medianas y rangos intercuartilos (RIC), según su distribución. Se utilizaron pruebas paramétricas y no paramétricas, según corresponda. Las diferencias se consideraron significativas con valores de $\mathrm{p}<0.05$.

\section{Resultados}

Cincuenta pacientes fueron incluidos en el estudio. Treinta y cuatro pacientes (68\%) eran hombres. La edad media fue de 65 años (DE \pm 12 ). En el grupo A (ACV crónico), se evaluaron 19 casos y en el grupo B (ACV agudo) 31 casos. En el Grupo A, la mediana de NIHSS en el momento de la evaluación fue de 2 (RIC 0-2) y la mediana de mRS fue de 1 . En el Grupo B, la mediana de NIHSS fue de 3 (RIC 2-5). El factor de riesgo vascular más frecuente fue la hipertensión (62\%), seguido del tabaquismo (22\%) y la dislipidemia (22\%).

En pacientes con ACV crónico, PLACS fue positiva en 4 pacientes (21\%) y CNS-LS fue positiva en 11 pacientes $(58 \%)$. Ambas escalas fueron positivas en 2 pacientes (10.5\%), y uno de ellos tuvo un IDB positivo. En este grupo, sólo un paciente cumplió con los criterios de diagnóstico de SPB propuestos (Figura 1).
En pacientes con ACV agudo, PLACS fue positiva en 9 pacientes (29\%) y CNS-LS fue positiva en 8 pacientes (26\%). Ambas escalas fueron positivas en 6 pacientes (19\%), y dos de ellos tuvieron un IDB positivo. En este grupo, cuatro pacientes cumplieron los criterios de diagnóstico de SPB propuestos (Figura 1).

En pacientes con SPB, la etiología más frecuente fue la aterosclerosis de grandes vasos y el 75\% de ellos tuvo un ACV en el hemisferio derecho. No se encontraron diferencias estadísticas con respecto al sexo, edad, tipo de ACV o NIHSS entre pacientes con o sin SPB.

\section{Discusión}

Diagnosticamos SPB en un paciente con ACV crónico y cuatro pacientes con ACV agudo.

El SPB es una condición definida por la existencia de episodios involuntarios o exagerados de expresión emocional de risa o llanto, que representan un cambio en la reactividad emocional posterior a una enfermedad específica. Estas respuestas son inconsistentes o desproporcionadas en relación con el estímulo, y no hay una mejor explicación de los eventos (como el consumo de sustancias o drogas ilegales, enfermedades psiquiátricas o deterioro cognitivo severo) (3). Afecta la calidad de vida de los pacientes y sus cuidadores.

Esta condición clínica puede estar presente en varias enfermedades neurológicas, incluidas la esclerosis lateral amiotrófica, la esclerosis múltiple y el trauma. En el $\mathrm{ACV}$, se ha reportado, según diferentes series, entre un 11 y un 34\% de los pacientes. En nuestra población, esta entidad está subdiagnosticada. Un estudio realizado en 2014 en nuestro hospital, en busca de SPB en diferentes patologías neurológicas, encontró una frecuencia de SPB en el ACV del 35\% (5).

Figura 1. Escalas realizadas en pacientes con ACV para búsqueda de sindrome pseudobulbar

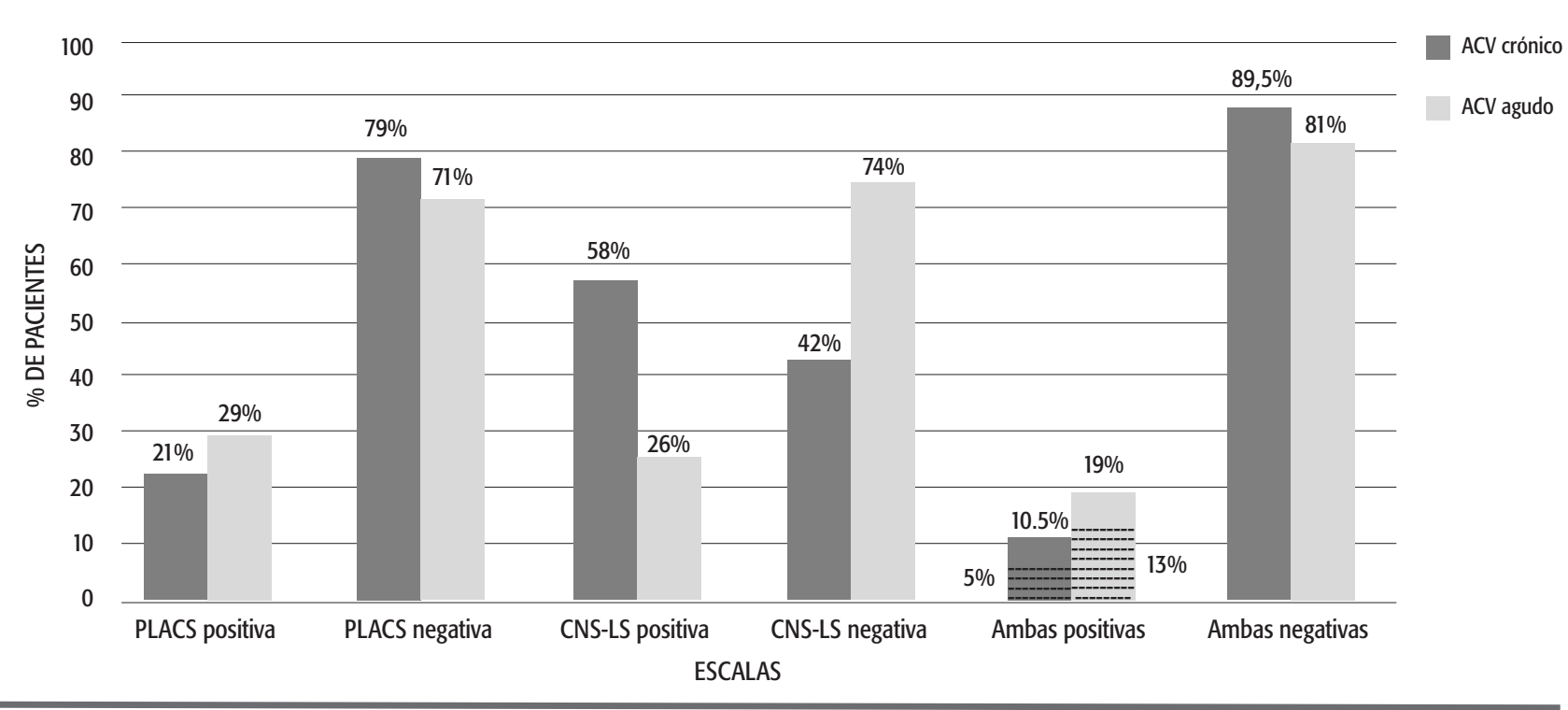

Se visualizan las escalas realizadas. La zona rayada representa los pacientes con diagnóstico de síndrome pseudobulbar (ambas escalas positivas y diagnóstico negativo de depresión por Inventario de Beck). 
Una revisión sistemática reciente y un meta-análisis de la literatura encontraron que el SPB es común después del ACV. El análisis de datos de 15 estudios reveló que 1 de cada 5 pacientes experimenta SPB dentro del primer mes del ACV, con una proporción similar a 6 meses después del ACV y 1 de cada 8 pacientes más allá de este punto (6).

Existen escalas autoadministradas que se han utilizado para objetivar y facilitar el diagnóstico de esta entidad, incluida la escala PLACS y la CNS-LS. En 1993, la escala PLACS fue publicada y validada para SPB en pacientes con ACV agudo. Tiene una sensibilidad del 88\% y una especificidad del 96\% para el diagnóstico (7). En 1997, la CNS-LS fue validada para la esclerosis lateral amiotrófica y en 2004 para la esclerosis múltiple. La sensibilidad y la especificidad reportadas son $84 \%$ y $81 \%$, respectivamente (8). Debido a su facilidad de uso, decidimos aplicar ambas escalas en nuestro estudio. Existen otras escalas para evaluar el SPB, como la Encuesta de Salud de Formulario Corto de 36 ítems (SF-36), el Cuestionario de 10 ítems del Centro de Estudios Epidemiológicos de Depresión (CES-D10) o el Cuestionario de Productividad Laboral y Deterioro de la Actividad (WPAI) (9).

El SPB se debe a la desregulación de 3 vías neurotransmisoras principales, dopamina, serotonina y glutamato, desde los lóbulos corticales frontales a través del cerebelo y el tronco del encéfalo, conocida como red córtico-límbico-subcorticotalámico-ponto-cerebelosa (10). El ACV, por daño directo o bien mediante la interrupción de los circuitos que se proyectan hacia el cerebelo y el tronco encefálico, puede provocar la desinhibición de las emociones voluntarias, haciéndolas involuntarias (9).

La combinación de dextrometorfano y quinidina es actualmente el único tratamiento aprobado por la FDA para el SPB. En Europa obtuvo autorización por la Agencia Europea de Medicamentos en 2013, pero fue retirado de mercado en 2016 por decisión de la empresa farmacéutica a cargo. El dextrometorfano actúa sobre los receptores sigma-1 y NMDA en el cerebro, y el sulfato de quinidina permite que el dextrometorfano alcance concentraciones terapéuticas (11). Hemos utilizado este tratamiento en el paciente con ACV crónico y SPB (sin depresión), con modestos beneficios a los seis meses de seguimiento.

Las tasas de diagnóstico de SPB han aumentado en los últimos años, posiblemente debido a un mejor reconocimiento, comprensión diagnóstica y disponibilidad de tratamiento (12). Después de nuestro estudio prospectivo, mantuvimos una administración estandarizada de ambas escalas (PLACS y CNS-LS) en la entrevista de admisión en la clínica ambulatoria de ACV.

Este estudio tiene algunas limitaciones. Primero, el grupo de pacientes es pequeño y se requiere un mayor número de casos para confirmar estos resultados. En segundo lugar, cuatro pacientes fueron diagnosticados en la etapa aguda del ACV. Es importante enfatizar que las condiciones inherentes a la hospitalización podrían tener un impacto en los resultados de las escalas. Por último, dado que no hay criterios de diagnóstico estandarizados, algunos pacientes pueden no haber cumplido con nuestros criterios propuestos y ser casos falsos negativos.

El SPB es una condición subdiagnosticada y no tratada, que complica al ACV tanto en la etapa aguda o crónica. Se desconoce la verdadera prevalencia de SPB en Argentina. La depresión se asocia con frecuencia y debe descartarse, ya que esta población podría beneficiarse de alternativas terapéuticas distintas a los antidepresivos. La labilidad emocional atribuible al SPB debe evaluarse cuidadosamente a través de preguntas exhaustivas al paciente, la familia y los cuidadores. Se deben aplicar al menos dos escalas para mejorar la sensibilidad diagnóstica.

\section{Declaración de conflictos de intereses}

Los autores no presentan conflictos de intereses respecto de este artículo.

\section{Referencias bibliográficas}

1. Thrift AG, Thayabaranathan T, Howard G, Howard VJ, Rothwell PM, Feigin VL, et al. Global stroke statistics. Int J Stroke 2017.12(1):13-32.

2. Schneider MA, Schneider MD. Pseudobulbar Affect: What Nurses, Stroke Survivors, and Caregivers Need to Know. J Neurosci Nurs 2017.49(2):114-7.

3. Girotra T, Lowe F, Feng W. Pseudobulbar affect after stroke: a narrative review. Top Stroke Rehabil. 2018;25(8):610-6.

4. Miller A, Pratt H, Schiffer RB. Pseudobulbar affect: The spectrum of clinical presentations, etiologies and treatments. Expert Rev Neurother. 2011;11(7):1077-88.
5. Quarracino C, Susana N, Arakaki T, Franco A, González L, Bohorquez N, et al. Neurol Arg. 2014;6(3):142-8.

6. Gillespie DC, Cadden AP, Lees R, West RM, Broomfield NM. Prevalence of Pseudobulbar Affect following Stroke: A Systematic Review and Meta-Analysis. J Stroke Cerebrovasc Dis. 2016;25(3):688-94.

7. Robinson G, Parikh M, Lipsey JR, Starkstein SE, Price R. Pathological laughing and crying following stroke: validation of a measurement scale and a double-blind treatment study. Psychiatry Interpers Biol Process. 1993;286-93.

8. Kasarkis EJ, Smith RA, Moore SR, Gresham LS, Bromberg MB. A self report measure of affective lability. I Neurol Neurosurg Psychiatry. 2008;63(1):89-93. 
9. Lapchak PA. Neuronal Dysregulation in Stroke-Associated Pseudobulbar Affect (PBA): Diagnostic Scales and Current Treatment Options. J Neurol Neurophysiol. 2015;06(05).

10. Parvizi J, Coburn KL, Shillcutt SD, Coffey CE, Lauterbach EC, Mendez MF. Neuroanatomy of Pathological Laughing and Crying: A Report of the American Neuropsychiatric Association Committee on Research. J Neuropsychiatry Clin Neurosci. 2014;21(1):75-87.
11. Zorowitz RD, Alexander DN, Formella AE, Ledon F, Davis C, Siffert J. Dextromethorphan/Quinidine for Pseudobulbar Affect Following Stroke: Safety and Effectiveness in the PRISM II Trial. PM R. 2018; S1934-1482(18)30352-6.

12. Towfighi A, Ovbiagele B, El Husseini N, Hackett ML, Jorge RE, Kissela BM, et al. Poststroke Depression: A Scientific Statement for Healthcare Professionals from the American Heart Association/American Stroke Association. Stroke.2017;48(2):e30-43. 